\title{
26
}

\section{INTERACTIVE RELIABILITY-BASED OPTIMAL DESIGN}

\author{
J.D. Sørensen*, P. Thoft-Christensen*, A. Siemaszko**, J.M.B. Cardoso** \& \\ J.L.T. Santos** \\ ${ }^{*}$ : CSR, Aalborg, Denmark $\quad{ }^{* *}$ : IST, IDMEC, Lisboa, Portugal
}

\begin{abstract}
Interactive design/optimization of large, complex structural systems is considered. The objective function is assumed to model the expected costs. The constraints are reliabilitybased and/or related to deterministic code requirements. Solution of this optimization problem is divided in four main tasks, namely finite element analyses, sensitivity analyses, reliability analyses and application of an optimization algorithm. In the paper it is shown how these four tasks can be linked effectively and how existing information on design variables, Lagrange multipliers and the Hessian matrix can be used in interactive optimization.
\end{abstract}

\section{Introduction}

Reliability-based optimal design of large, complex structural and mechanical systems, e.g. aerospace structures is considered. Uncertain quantities are modelled by stochastic variables and the reliability is estimated using First Order Reliability Methods, see Madsen et. al [1]. Sensitivity information is obtained using the adjoint continuum method of design sensitivity analysis, see Haug et. al [2]. An optimization problem is considered where the design (optimization) variables are assumed to be related to size variables defining the geometry of the structural elements and to shape variables defining the overall geometry of the structure.

Reliability-based optimization of structural systems has been considered in a number of papers, see e.g. Murotsu et. al [3], Frangopol [4], Sørensen \& Thoft-Christensen [5] and Enevoldsen \& Sørensen [6]. A reliability-based optimization problem can be formulated as minimization of e.g. the structural weight with constraints on element reliability indices and/or on the system reliability. Alternatively the total expected costs in the design lifetime can be minimized or a multiobjective optimization problem can be formulated.

The objective function can in this paper model the expected costs related to the structure in its planned lifetime or it can simply be the volume of the structure. Deterministic constraints can be imposed to ensure that certain response characteristics such as displacements and stresses do not exceed codified critical values. The deterministic constraints can also include general design requirements for the design variables. Further constraints are included to ensure that the reliability of the structure is satisfactory, see section 2 . 
Special emphasis is given to techniques to perform the optimization interactively, see section 3. Basic interactive operations are described and it is shown how existing information on design variables, Lagrange multipliers and the Hessian matrix can be used in interactive optimization.

Solution of this optimization problem can be divided in four main tasks, namely finite element analyses, sensitivity analyses, reliability analyses and application of an optimization algorithm. For realistic structures solution of a reliability-based optimization problem can be very computertime demanding. It is shown how these four tasks performed by individual computer programs can be linked effectively, see section 4. An example with size and shape variables is shown, see section 5 . Special effort is given to demonstrate the effect of taking into account the available information in interactive optimization.

\section{General formulation of optimization (design) problem}

The following general reliability-based structural optimization problem is considered

$$
\begin{array}{ll}
\min & C(\mathbf{b})=\sum_{i=1}^{m_{D}} C_{I_{i}} V_{i}(\mathbf{b})+\sum_{i=1}^{m_{P}} C_{f_{i}} \Phi\left(-\beta_{i}(\mathbf{b})\right) \\
\text { s.t. } & \beta_{i}(\mathbf{b}) \geq \beta_{i}^{\text {min }} \quad, i=1, \ldots, M \\
& B_{i}(\mathbf{b}) \geq 0 \quad, i=1, \ldots, m \\
& b_{i}^{l} \leq b_{i} \leq b_{i}^{u} \quad, i=1, \ldots, N
\end{array}
$$

where $\mathbf{b}=\left(b_{1}, \ldots, b_{N}\right)$ are the design (or optimization) variables. The optimization variables are assumed to be related to parameters defining the geometry of the structure (for example diameters and thicknesses of tubular elements) and coordinates (or related parameters) defining the geometry (shape) of the structural system.

The objective function $C$ consists of a deterministic and a probabilistic part with $m_{D}$ and $m_{P}$ terms, respectively. $V_{i}$ is e.g. a volume in the $i$ th deterministic term and $C_{I_{i}}$ is the cost per volume of the $i$ th term modelling the construction costs. $V_{i}$ is assumed to be deterministic. If stochastic variables influence $V_{i}$ then design values, see below, are assumed to be used to calculate $V_{i}$. In the probabilistic part $C_{f_{i}}$ is the cost due to failure of failure mode $i . \Phi$ is the standard Normal distribution function and $\beta_{i}, i=1, \ldots, m_{P}$ are reliability indices for $m_{P}$ failure modes. The general formulation of (1) allows the objective function to model both the structural weight and the total expected costs of construction and failure.

The constraints in (2) are based on the reliability indices $\beta_{i}, i=1, \ldots, M$ for $M$ failure modes. $\beta_{i}^{\text {min }}, i=1, \ldots, M$ are the corresponding lower limits on the reliabilities. $B_{i}, i=1, \ldots, m$ define the deterministic inequality constraints in (3) which can ensure that response characteristics such as displacements and stresses do not exceed codified critical values. Determination of the inequality constraints usually includes finite element analyses of the structural system. The inequality constraints can also include general design requirements for the design variables. The constraints in (4) are so-called simple 
bounds.

The variables (parameters) used to model the structure to be analysed are characterized as stochastic or deterministic if the variable can be modelled as stochastic or deterministic and design or fixed if the variable can be a design (optimization) variable or a fixed constant.

The stochastic variables are denoted $\mathbf{X}$ and deterministic variables are denoted $\mathbf{x}^{D}$. The design variables $\mathbf{b}$ can be expected values of stochastic variables or deterministic variables. The reliability indices in (1) and (2) are determined on basis of limit state functions

$$
g_{i}\left(\mathbf{x}(\mathbf{b}), \mathbf{x}^{D}(\mathbf{b}), \mathbf{Y}\left(\mathbf{x}(\mathbf{b}), \mathbf{x}^{D}(\mathbf{b})\right)\right)=0 \quad i=1, \ldots, M
$$

where $\mathbf{x}$ are realisations of the stochastic variables $\mathbf{X}$. $\mathbf{Y}$ are performances such as displacements and stresses calculated by structural analysis, see section 4 . The deterministic constraints in (3) are assumed to be related to the limit state functions $g_{j}$ by

$$
B_{j}=g_{j}\left(\mathbf{x}^{d}(\mathbf{b}), \mathbf{x}^{D}(\mathbf{b}), \mathbf{Y}\left(\mathbf{x}^{d}(\mathbf{b}), \mathbf{x}^{D}(\mathbf{b})\right)\right) \quad j=1, \ldots, m
$$

$x_{i}^{d}$ is a design value calculated from $x_{i}^{d}=\gamma_{i}\left(\mu_{X_{i}}+k_{i} \sigma_{X_{i}}\right)$ where $k_{i}$ is a factor defining the characteristic value and $\gamma_{i}$ is a partial safety factor. $\mu_{X_{i}}$ and $\sigma_{X_{i}}$ are expected value and standard deviation of $X_{i}$, respectively.

\section{Interactive optimization}

The basic types of interactive optimization which influences the formulation of the optimization problems are, see Arora [7]: • include (delete) a design (optimization) variable, - include (delete) a constraint, $\bullet$ modify a constraint or $\bullet$ modify (change) the objective function.

In order to investigate the effect of interactive optimization on the optimality criteria (1) - (4) is restated as an optimization problem with objective function $C(\mathbf{b})$, equality constraints $c_{i}(\mathbf{b})=0, i=1, \ldots, m_{e}$ and inequality constraints $c_{i}(\mathbf{b}) \geq 0, i=m_{e}+$ $1, \ldots, m$. First order necessary conditions that have to be satisfied at a (local) optimum point $\mathbf{b}^{*}$ are given by the Kuhn-Tucker conditions. If the optimization process has almost converged, a good guess on the optimal design is available. A modification of the optimization problem is then specified by the user. The influence on the optimality criteria is described in the following, see Sørensen [8] for details.

Include/delete an optimization variable: Let one of the fixed parameters $p$ in the model of the structural system be transferred from the list of fixed parameters to the list of optimization variables. Let the new optimization variable be denoted $b_{N+1}$. The new optimal point is then denoted $\mathbf{b}^{+}=\left(b_{1}^{+}, \ldots, b_{N}^{+}, b_{N+1}^{+}\right)$and the Kuhn-Tucker conditions 
are modified to

$$
\begin{array}{rlrl}
\frac{d C\left(\mathbf{b}^{+}\right)}{d b_{j}^{+}}-\sum_{i=1}^{m} \lambda_{i}^{+} \frac{d c_{i}\left(\mathbf{b}^{+}\right)}{d b_{j}^{+}} & =0 & & , j=1,2, \ldots, N+1 \\
\lambda_{i}^{+} c_{i}\left(\mathbf{b}^{+}\right)=0 \quad, \lambda_{i}^{+} \geq 0 & & , i=m_{e}+1, \ldots, m \\
c_{i}\left(\mathbf{b}^{+}\right)=0 & , i=1, \ldots, m_{e} \\
c_{i}\left(\mathbf{b}^{+}\right) \geq 0 & , i=m_{e}+1, \ldots, m
\end{array}
$$

where $\lambda_{i}^{+}, i=1, \ldots, m$ are the multipliers corresponding to the optimum point. Compared with the unmodified case an extra equation is introduced in (7). The variables in the modified optimum point $\mathbf{b}^{+}$can be expected to be very close to the point $\left(\mathbf{b}^{*}, p\right)$ because all the constraints in (8)-(10) are satisfied with $\mathbf{b}^{*}$ and the parameter $p$. Also the first $N$ equations in $(7)$ are satisfied in the point $\left(\mathbf{b}^{*}, p\right)$. If optimization variable $b_{N}$ is deleted from the list of optimization variables and included as a parameter $p$ then the new optimal point $\left(\mathbf{b}^{-}, p\right)$ can also be expected to be very close to $\mathbf{b}^{*}$.

The number of Lagrangian multipliers $\boldsymbol{\lambda}^{+}$is unchanged and the numerical values can be expected to be almost unchanged. If an optimization variable is included then the size of the Hessian matrix $\mathbf{H}$ of the Lagrangian function (which is used in some optimization algorithms) is increased with one compared with the old Hessain matrix $\mathbf{H}^{\text {old }}$. A first estimate of the updated Hessian matrix is $\mathbf{H}=\left[\begin{array}{cc}\mathbf{H}^{\text {old }} & 0 \\ 0^{T} & 1\end{array}\right]$ where $0^{T}=(0,0, \ldots, 0)$. If an optimization variable is deleted, the size of the Hessian matrix is decreased with one. A first estmate of the updated Hessian matrix is simply to delete the row and column corresponding to the deleted optimization variable. The updated Hessian matrix can be used as input to restarted optimization algorithms.

Include/delete a constraint : Let a new inequality constraint be introduced as constraint no. $m+1$. The new optimal point is denoted $\mathbf{b}^{+}=\left(b_{1}^{+}, \ldots, b_{N}^{+}\right)$. The number of equations in (8) and (10) is increased by one and a new multiplier $\lambda_{m+1}^{+}$is introduced. The new optimum $\mathbf{b}^{+}$can be quite different from $\mathbf{b}^{*}$, depending on the new constraint $c_{m+1}$. The size of the Hessian matrix is not changed and a first estimate of $\mathbf{H}$ can simply be the old Hessian matrix. If a constraint is deleted then the same considerations as for inclusion of a constraint can be stated. If the new constraint or the deleted constraint is not active then the optimality criteria is satisfied with $\mathbf{b}^{*}$.

Modify a constraint : Let the constraint no. $m$ be modified to $c_{m}^{\prime}(\mathbf{b})$. The new optimal point is denoted $\mathbf{b}^{+}=\left(b_{1}^{+}, \ldots, b_{N}^{+}\right)$. In this case the number of equations is unchanged but the new optimum $\mathbf{b}^{+}$can be quite different from $b^{*}$, depending on the modified constraint $c_{m}$. The size of the Hessian matrix is also unchanged and a first estimate of $\mathbf{H}$ can simply be the old Hessian matrix.

Modify the objective function: The objective function in (1) can be modified by changing the performances in the deterministic part, the failure modes in the probabilistic part or by changing the cost factors $C_{I_{i}}$ and $C_{f_{i}}$. Modification of the objective function in (1) has almost the same effect on the Kuhn-Tucker conditions as modification of a constraint. 


\section{Integrated optimization techniques}

The main modules in the intergrated optimization system CARBOS, [10] are OPT : Reliability-based interactive optimization by RELOPT, [11], DSA : Design sensitivity analysis by the continuum method, [12], FEA : Finite element calculations by ASASNL, [13], REL : Reliability analysis by RELIAB, [14] or COMREL, [15] and UI : User interface module which generates ASCII files with interactive input to OPT, REL and FEA. The data flow is shown in figure 1.

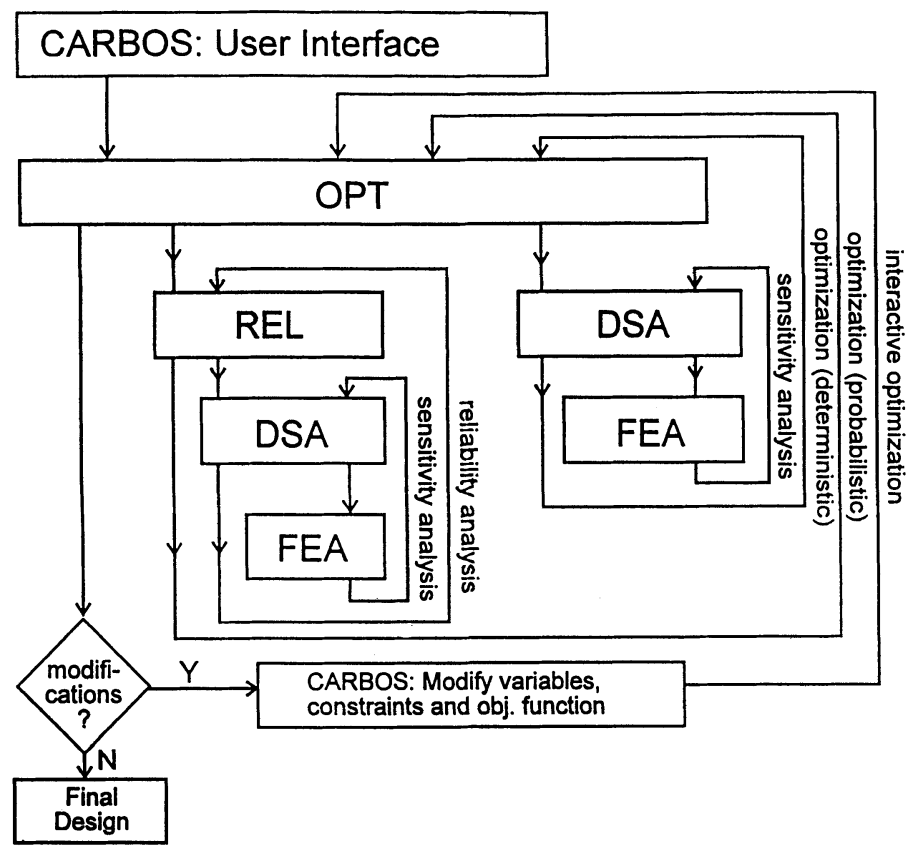

Figure 1. Data flow in interactive optimization by CARBOS.

\section{Example}

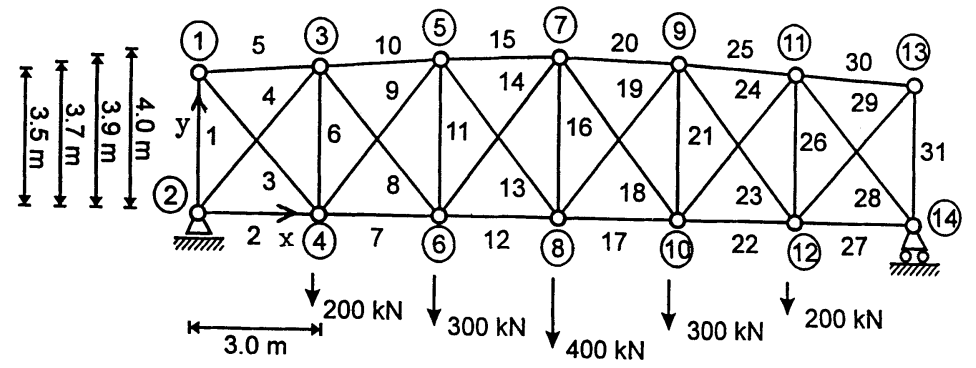

Figure 2. Bridge consisting of 31 elements and 14 nodes. 
A simple 2D truss model of a bridge structure is considered, see figure 2. The truss elements are all tubular elements and are divided into three groups with identical diameters. The bottom truss elements $(2,7,12,17,22$ and 27$)$ are group 1 , the top truss elements $(5,10,15,20,25$ and 30$)$ and the two outer diagonals ( 3 and 29) are group 2 and the remaining are belonging to the $3 \mathrm{rd}$ material group.

Reliability model : The stochastic variables used are shown in table 1, where SIGMAX models the maximun acceptable stress $\sigma_{\max }$ and ADM models the maximun acceptable displacement $u_{\max }$.

Table 1. Stochastic variables. $\mu$ : expected value, $\sigma$ : standard deviation. N: normal, LN: lognormal, G: Gumbel. $b_{1} \ldots b_{10}$ are design variables.

\begin{tabular}{lcll} 
& distribution & $\mu$ & \multicolumn{1}{c}{$\sigma$} \\
\hline Load multiplier & $\mathrm{G}$ & 1 & 0.40 \\
Diameter group 1 & $\mathrm{N}$ & $b_{1}$ & $0.1 b_{1}$ \\
Diameter group 2 & $\mathrm{N}$ & $b_{2}$ & $0.1 b_{2}$ \\
Diameter group 3 & $\mathrm{N}$ & $b_{3}$ & $0.1 b_{3}$ \\
$y$-coor. node 1,3,5,7,9,11 and 13 & $\mathrm{N}$ & $b_{4}, \ldots, b_{10}$ & $50 \mathrm{~mm}$ \\
SIGMAX & $\mathrm{LN}$ & $300 \mathrm{~N} / \mathrm{mm}^{2}$ & $30 \mathrm{~N} / \mathrm{mm}^{2}$ \\
ADM & $\mathrm{N}$ & $45 \mathrm{~mm}$ & $4.5 \mathrm{~mm}$ \\
\hline
\end{tabular}

Optimization problem : To specify the optimization problem 19 limit states are defined. The limit state functions are

$$
\begin{aligned}
& g_{1}=\mathrm{ADM}-\mathrm{PER} 2 \\
& g_{j}=\operatorname{SIGMAX}-\operatorname{PER}(j+1) \quad, j=2, \ldots, 19
\end{aligned}
$$

The optimization variables are $\mathbf{b}=\left(d_{1}, d_{2}, d_{3}, y_{1}, y_{3}, y_{5}, y_{7}, y_{9}, y_{11}, y_{13}\right)$, see table 1 . The initial values are $\mathbf{b}=(150,150,150,3500,3700,3900,4000,3900,3700,3500)[\mathrm{mm}]$. The objective function is $C(\mathbf{b})=$ Volume and the constraints are $\beta_{1} \geq 3.29$ and $B_{j}=g_{j} \geq 0, j=$ $2,3, \ldots, 19 . \beta_{1}$ is calculated using $g_{1}$. In the deterministic constraints the design values are calculated using $k=-2$ and $\gamma=0.8$ for SIGMAX, $k=2$ and $\gamma=1.2$ for the load and $k=0$ and $\gamma=1.0$ for all other parameters. The reliability analyses are performed using RELIAB [14]. The optimization problem is solved using RELOPT [11] and the NLPQL optimizer [9] with convergence limit $\epsilon=10^{-7}$.

Interactive optimization results : The optimization problem is solved interactively in three main steps where interaction is possible for each 4 iterations:

1. The optimization problem is solved with only the reliability constraint included.

First 4 iterations are performed with the intitial values of the optimization variables as start point. Next restart is done where the 'old' Hessian matrix and Lagrange multipliers are used. 4 iterations is performed. This restart procedure is repeated until convergence.

2. Deterministic constraints are included and optimization is continued with $2 \mathrm{a}$ or $2 \mathrm{~b}$ :

2a. All 'old' values of design variables, Hessian matrix and Lagrange multipliers are used as start conditions. This restart procedure is repeated in blocks of 4 iterations until convergence. 
2b. First 4 iterations are performed where only the current values of the optimization variables are used as starting info. Next restarts are performed as in 2a until convergence.

3. The diameters of the struss elements are fixed on reasonable values and unimportant stress constraints are deleted. The optimization is continued with $3 \mathrm{a}$ or $3 \mathrm{~b}$ :

3a. All 'old' values of design variables, Hessian matrix and Lagrange multipliers are used as start conditions. This restart procedure is repeated in blocks of 4 iterations until convergence.

3 b. First 4 iterations are performed where only the current values of the optimization variables are used as starting info. Next restarts are performed as in $2 \mathrm{a}$ until convergence.

The initial volume is $C(\mathbf{b})=21.210^{8}$. The results of the optimization are:

Step 1: $\mathbf{b}=\left(d_{1}, d_{2}, d_{3}, y_{1}, y_{3}, y_{5}, y_{7}, y_{9}, y_{11}, y_{13}\right)=(102.1,108.4,51.5,2000,3357,4759$, $5000,4759,3357,2000), C(\mathbf{b})=5.6610^{8}$.

Step 2: $\mathbf{b}=\left(d_{1}, d_{2}, d_{3}, y_{1}, y_{3}, y_{5}, y_{7}, y_{9}, y_{11}, y_{13}\right)=(103.3,110.0,51.7,3102,3389,4806$, $5000,4806,3389,3102), C(\mathbf{b})=5.8210^{8}$.

Step 3: $\mathbf{b}=\left(d_{1}, d_{2}, d_{3}, y_{1}, y_{3}, y_{5}, y_{7}, y_{9}, y_{11}, y_{13}\right)=(110$ (fixed), 110 (fixed), 60 (fixed), $2000,3234,4835,5000,4835,3234,2000), C(\mathbf{b})=6.5210^{8}$.

To measure the computation time the number of finite element analyses of the structure is counted. This number can be considered as a good indication of the computational effort. The results are shown in table 2. It is seen that in this example the amount of computations is decreased if information in the form of Lagrange multipliers and Hessian matrices are used when the optimization problem is restartet after a modification. The optimized structure is shown in figure 3 .

Table 2. Total number of finite element analyses using the different four strategies.

\begin{tabular}{ccccc}
\hline & $1+2 \mathrm{a}+3 \mathrm{a}$ & $1+2 \mathrm{a}+3 \mathrm{~b}$ & $1+2 \mathrm{~b}+3 \mathrm{a}$ & $1+2 \mathrm{~b}+3 \mathrm{~b}$ \\
\hline step 1 & 59 & 59 & 59 & 59 \\
step 2 & 76 & 76 & 111 & 111 \\
step 3 & 96 & 109 & 125 & 144 \\
\hline
\end{tabular}

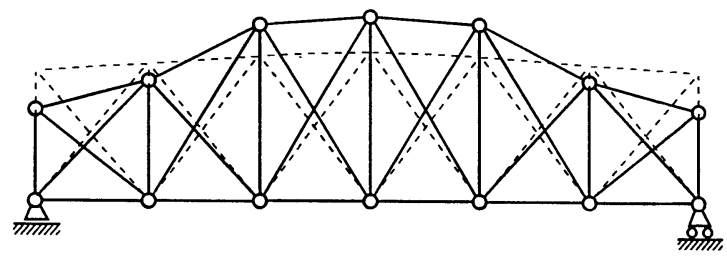

Figure 3. Initial (dashed lines) and optimized structure.

\section{Conclusions}

A general reliability-based optimization problem for interactive design/optimization of large, complex structural systems is described. Techniques for interactive solution of this 
optimization problem are discussed, especially how to use information from Lagrange multipliers and from the Hessian matrix. Integration of the main tasks in solution of this optimization problem, finite element analyses, sensitivity analyses, reliability analyses and application of an optimization algorithm is discussed briefly. An example illustrates the interactive optimization.

\section{Acknowledgements}

The paper presents parts of results of the BRITE/EURAM P4194 Project "Reliability Based Optimal Design with Applications to Advanced Aerospace Structures". This project, which started in September 1991, is funded through a $50 \%$ contribution from the Directorate General for Science, Research and Development of the Commision of the European Communities and another $50 \%$ contribute from the partners in the project: • WS Atkins Science and Technology, Epsom, UK, • Computational Safety and Reliability (CSR), Aalborg, Denmark, • Instituto Superior Tecnico, Lisbon, Portugal, • MAN Technologie, Munich, Germany and • Reliability Consulting and Programs (RCP), Munich, Germany.

\section{References}

[1] Madsen, H.O., S. Krenk \& N.C. Lind: Methods of Structural Safety. Prentice-Hall, 1986.

[2] Haug, E.J., K.K. Choi \& V. Komkov: Design Sensitivity Analysis of Structural Systems. Academic press, 1986.

[3] Murotsu, Y., M. Kishi, H. Okada, M. Yonezawa \& K. Taguchi: Probabilistically Optimum Design of Frame Structures. Proc. 11th IFIP Conf. on "System Modelling and Optimization". Springer Verlag, 1984, pp. 545-554.

[4] Frangopol, D.: Sensitivity of Reliability-Based Optimum Design. ASCE, Journal of Structural Engineering, Vol. 111, No. 8, 1985, pp. 1703-1721.

[5] Sørensen, J.D. \& P. Thoft-Christensen: Structural Optimization with Reliability Constraints. Proc. 12th IFIP Conf. on "System Modelling and Optimization". Springer Verlag, 1985, pp. 876-885.

[6] Enevoldsen, I. \& J.D. Sørensen: Reliability-Based Optimization in Structural Engineering. Structural Safety, Vol. 15, 1994, pp. 169-196.

[7] Arora, J.S.: Introduction to Optimum Design. McGraw-Hill, 1989.

[8] Sørensen, J.D.: Optimization methods and interactive optimization. BRITE/EURAM P4194 report B(V.1) - 4.2, CSR, March 1994.

[9] Schittkowski, K.: NLPQL: A FORTRAN Subroutine Solving Constrained NonLinear Programming Problems. Annals of Operations Research, 1986.

[10] CARBOS: BRITE/EURAM P4194 report, WS Atkins, July 1994.

[11] RELOPT: RELiabilty-based OPTimization. CSR-Software, CSR, Aalborg, Denmark, August 1994.

[12] IST: Interactive Sensitivity Tools. User's guide, Version 2.0B, CEMUL, IST, Lisbon, 1993.

[13] ASASNL: User's Guide, Version 18, WS Atkins, UK, 1990.

[14] RELIAB: Reliabilty analysis. CSR-Software, CSR, Aalborg, Denmark, February 1994.

[15] COMREL-TI: User's manual. RCP GmbH, Munich, Gernany, 1992. 